\title{
Study on Strain Rate Effect and Size Effect of Dynamic Response Characteristics of Granite
}

\author{
Zhihang Hu*, Yuying Ning, Jiuyang Zhang, Jianyu Zhao \\ School of Mining Engineering, University of Science and Technology Liaoning, Anshan 114051, Liaoning, China \\ Engineering and Technology Research Center of High-efficiency Mining-processing and Utilization of Metal Mineral Resources of \\ Liaoning Province, Anshan 114051, Liaoning, China \\ Email: huzhihang123@foxmail.com
}

\begin{abstract}
Under impact load, the dynamic mechanical properties of rock are complex and changeable. The Split Hopkinson Pressure Bar (SHPB) system was used to change the impact load to carry out different strain rate loading tests on granite with different aspect ratios, and to analyze the influence of strain rate and aspect ratio on the dynamic energy consumption of granite crushing. The results show that at an impact velocity of $14 \mathrm{~m} / \mathrm{s}$, the granite with an aspect ratio of 1.4 appears to be strip-shaped fragments after being broken; the granite with an aspect ratio of 1.0 uniform square fragments after being broken; the granite with an aspect ratio of 0.6 appears to be a large number of flat fragments after being broken. When the load strain rate of the granite with an aspect ratio of 0.6 increases from $50 \mathrm{~s}^{-1}$ to $150 \mathrm{~s}^{-1}$, the energy-time density index increases significantly; when the load strain rate exceeds $150 \mathrm{~s}^{-1}$, the energy-time density index decreases. When the strain rate of granite with an aspect ratio of 1.0 exceeds $80 \mathrm{~s}^{-1}$, the energy-time density increases significantly. When the strain rate of the granite with an aspect ratio of 1.4 exceeds $60 \mathrm{~s}^{-1}$, the rate of increase of the energy-time density of the rock increases significantly.
\end{abstract}

Keywords: strain rate, fragmentation, SHPB, energy-time density

\section{Introduction}

When the traditional two-wave method is used to study the response of rock fragmentation during the SHPB test, it is assumed that the rock is in a uniform stress state. The stress balance process and the uniform duration of stress also have a strong size effect and strain rate effect, so the influence of the stress wave on the time distribution of the stress action during the propagation of the stress wave in the rock cannot be ignored. Li et al. ${ }^{[1,2]}$ studied the variation of the peak damage of brittle material specimens with age, and concluded that the internal damage and residual reflected waves of rock specimens are the main factors that cause subsequent failure of the specimens. Li Diyuan et al. ${ }^{[3]}$ used a traditional SHPB test device to perform impact tests on marble samples with prefabricated holes and found that it was related to the long axis of the hole, the loading direction and ratio of semi-minor axis length. Ping $\mathrm{Qi}^{[4]}$ and other studies found that the energy loaded in the test mainly acts on the deformation, failure and damage evolution of the specimen. Liu Xiaohui et al. ${ }^{[5,6]}$ used the stress wave theory to analyze the energy consumption of the coal sample and found that the energy dissipation is related to the crushing particle size. Li Yexue et al. ${ }^{[7,8]}$ found that the greater the fractal dimension of the fracture surface of jointed rocks, the greater the rate of energy dissipation. From the perspective of energy conversion, Liang Changyu et al. ${ }^{[9]}$ interpreted the cause of the size effect of granite under different strain rates, and concluded that the rock size is negatively correlated with the energy absorbed during rock failure and the elastic strain energy. In general, the process of energy dissipation is irreversible, while the release of energy is bidirectional and reversible under certain conditions. ${ }^{[10]}$

In order to eliminate the influence of the specimen size on the energy dissipation of the specimen, most scholars use the energy dissipation per unit volume to characterize the magnitude of the stress wave energy absorbed by the specimen. ${ }^{[1]}$ However, in addition to the size of the specimen, the structural characteristics of incident energy include two aspects. Based on this, Guo Lianjun ${ }^{[12]}$ defined the energy dissipation per unit volume per unit time as a new index for judging the energy dissipation of the specimen. Taking this as a starting point, the three-wave method is used to obtain effective data of stress wave propagation inside the rock; the whole impact process of the rock is evaluated with the matching state of stress balance; the energy consumption characteristics of crushing granites with different aspect ratios under different loading strain rates are studied; the dynamic response characteristics of the rock are analyzed. 


\section{Test plan design}

\subsection{Principles of test equipment}

The test was completed using the SHPB test system of Liaoning University of Science and Technology. The cylindrical punch, incident rod, transmission rod and buffer rod of the test device are all 50Cr steel, with a Young's modulus of $240 \mathrm{GPa}$, a density of $7800 \mathrm{~kg} / \mathrm{m}^{3}$, a longitudinal wave velocity of $5580 \mathrm{~m} / \mathrm{s}$ in the rod, and a wave impedance of $4.35 \times 10^{7} \mathrm{MPa} / \mathrm{s}$.
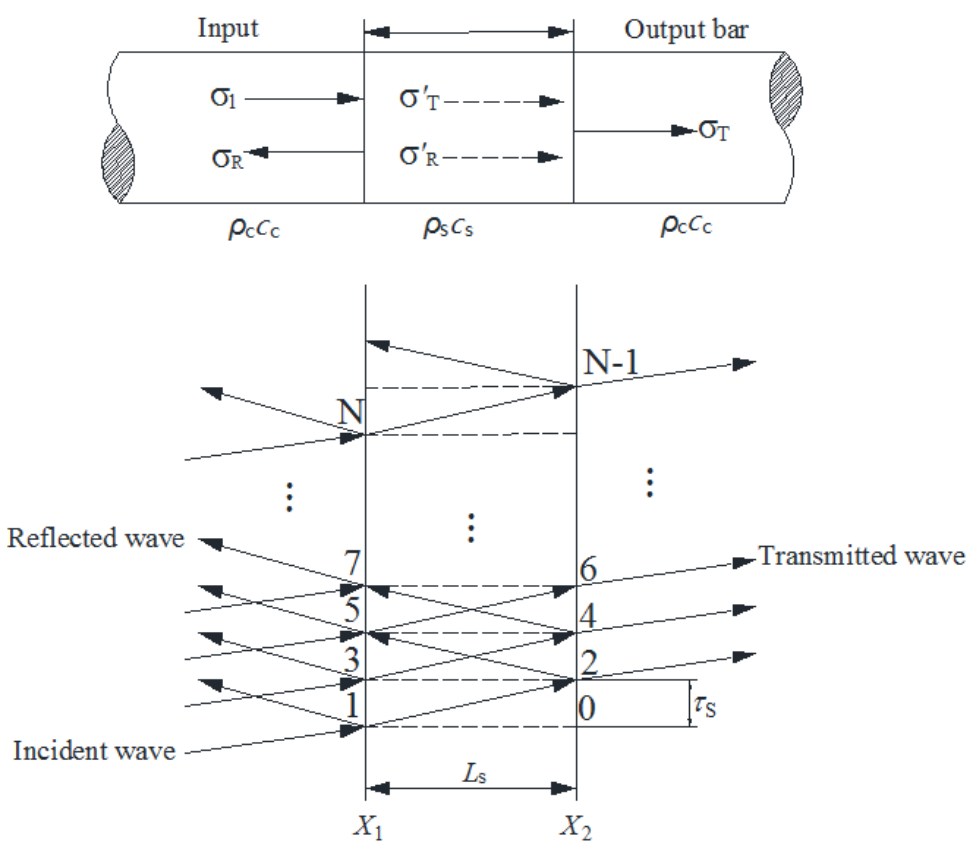

Figure 1. Schematic diagram of SHPB compression rod device ${ }^{[13]}$

\subsection{Test plan}

Increase the number of tests under the same conditions; ensure the consistency of impact strength through bullet speed; deal with the dispersion of test results; the total number of rock specimens is 60 times. The granite specimen is shown in Figure 2.

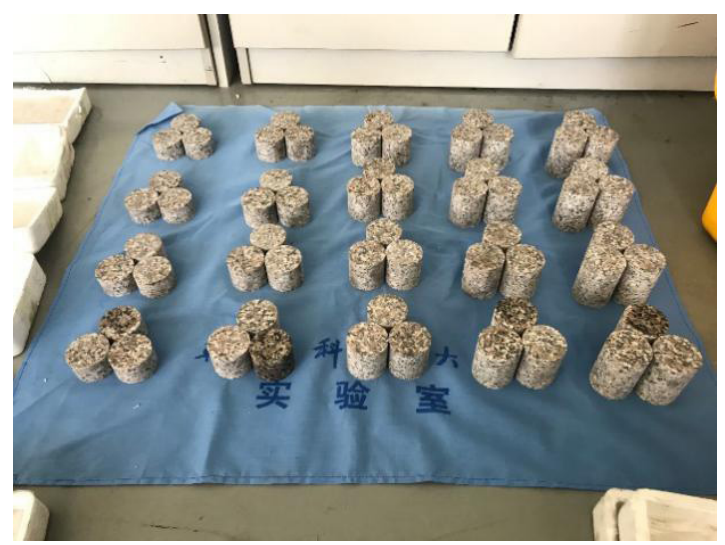

Figure 2. Granite specimens with five aspect ratios

Since the dynamic compressive strength of the rock increases with the increase in the aspect ratio, considering that the designed aspect ratio is more than 1 times the difference between the two boundary dimensions, the granite specimen with an aspect ratio of 1.0 was tested before the test. For the purpose of rationally optimizing the loading strain rate, the specimens with a aspect ratio of 0.6 and 1.4 were respectively loaded with the maximum and minimum values of the design air pressure. Effective data can be obtained while ensuring that most of the load strain rate ranges overlap. 


\section{The effect of stress balance on granite fractured morphology}

\subsection{The influence of strain rate on the stress-strain curve}

According to the voltage signals collected on the incident rod and the transmission rod, the data is processed by the three-wave method. A typical stress-strain curve is shown in Figure 3. Under the same impact velocity, granites with different aspect ratios have different response capabilities to dynamic loads. Among them, the peak stresses of 5 groups of tests are relatively close. According to the deformation and failure ability of the rock, it can be found that there is an obvious critical value for the aspect ratio, which makes the response modes of both ends different.

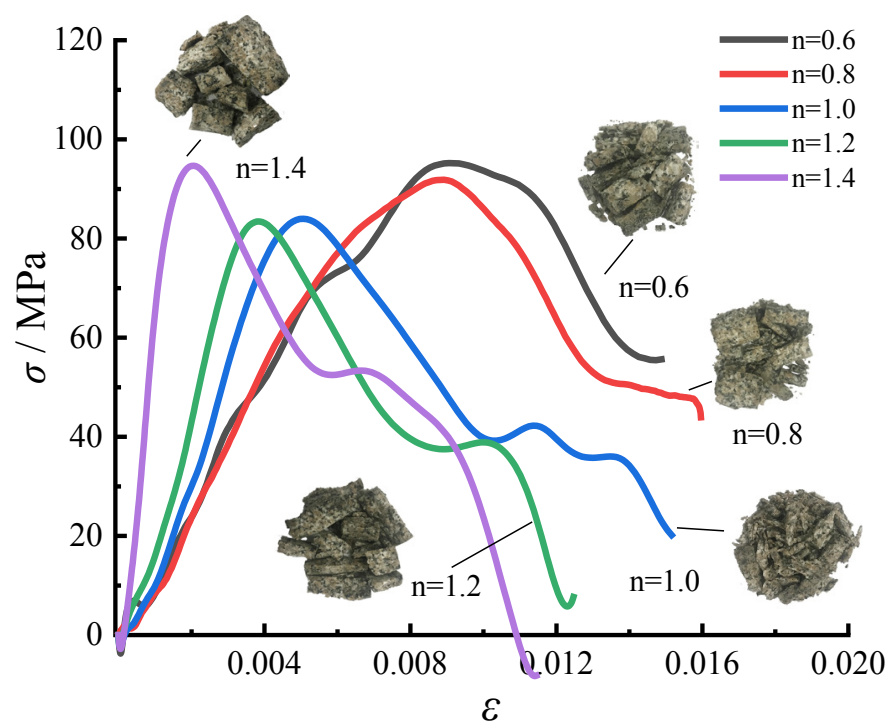

Figure 3. Strain-stress curves of granites with different aspect ratios

Judging from the microstructure characteristics of the stress-strain curve, granite originally belongs to the stage of elastic compression, making the $\sigma-\varepsilon$ curve approximate a linear rise. Under different strain rates, after the specimen stress rises to a certain value, the curve begins to become flat, that is, the slope of the curve gradually decreases. Before the stress of the specimen reaches the peak value, the curve fluctuates to varying degrees. This is because of the different duration of time for uniform stress in the rock and the uniform degradation of stress after the rock mass is deformed and destroyed. The declining form of the $\sigma-\varepsilon$ curve after the peak point is closely related to the strain rate, which characterizes the damage degree of the specimen. The area under the stress-strain curve is the total energy dissipated during dynamic compression. ${ }^{[14]}$ In this interval, as the strain rate increases, the ability of rock deformation accumulation increases, which increases the degree of granite fracture.

\subsection{Dynamic stress concentration area}

Figure 5 shows the broken shape of the rock with the aspect ratio reduced from 1.4 to 0.6 at an impact velocity of 14 $\mathrm{m} / \mathrm{s}$. It can be found from the figure that the granite with a aspect ratio of 1.4 is broken into long strips. When the aspect ratio of granite is 1.0 , the rock fragments are mostly uniform square fragments. When the aspect ratio decreases to 0.6 , a large number of flat fragments appear after the rock is broken. This shows that when the aspect ratio is 0.6 , under the action of $14 \mathrm{~m} / \mathrm{s}$ impact velocity, within the given stress pulse time, the uniform stress area has completely covered or even exceeded the rock itself, and that the part that overlaps with the rock is more the center of the uniform stress area.

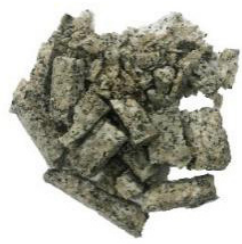

(a) The broken form of 1.4 aspect ratio

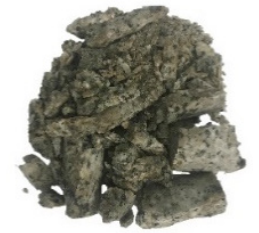

(b) The broken form of 1.0 aspect ratio

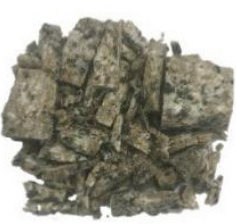

(c) The broken form of 0.6 aspect ratio

Figure 4. The impact of the reduced aspect ratio on the shape of rock fragmentation at an impact velocity of $14 \mathrm{~m} / \mathrm{s}$ 
In the same way, the radial end face of the rock also cracks from the central area. The stress concentration in the center area of the rock end face is faster than that in the boundary area. At the same time, when the stress wave propagates from the inside of the rock to the free surface of the side, it causes reflection and stretching, which promotes the propagation of radial cracks. The crack density of the rock is also controlled by the stress concentration area. Under the action of a small loading strain rate, the rock is more likely to show a fractured shape. With the increase of the strain rate, the radial cracks randomly spread to the surrounding defect areas that have been activated, making the rock fragmentation transition from large pieces to small pieces.

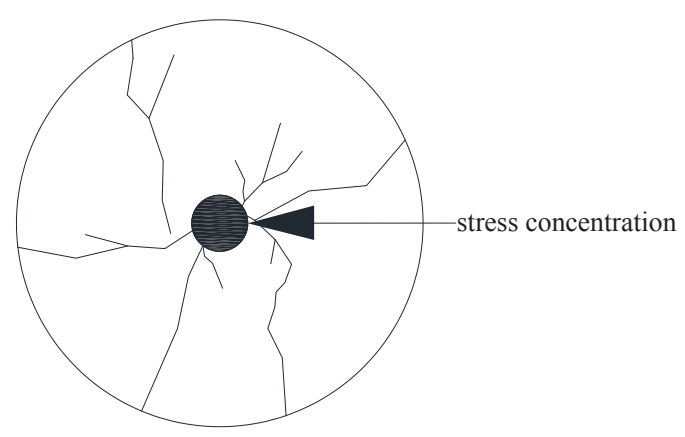

(a) Rock stress distribution in the early stage of dynamic load

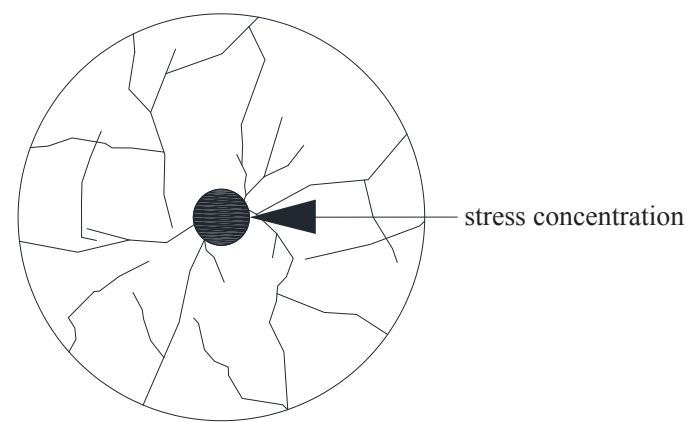

(b) Rock stress distribution in the later stage of dynamic load

Figure 5. Fractured shape of rock radial section

Figure 6 shows the radial fracture morphology with different aspect ratios at an impact velocity of $8 \mathrm{~m} / \mathrm{s}$. It can be seen from the cross section that the rock stress concentration area is located in the center of the rock end face. The rock cracks in the high stress concentration area spread from here to the surroundings. As the aspect ratio increases, the number of cracks in the rock decreases significantly. This is because the energy carried by the stress pulse makes the rock prone to fracture formation under the action of impact load.

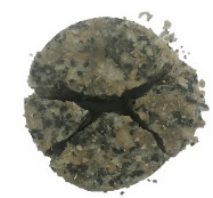

(a) $\mathrm{n}=0.6$

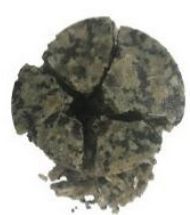

(b) $\mathrm{n}=0.8$

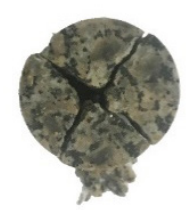

(c) $\mathrm{n}=1.0$

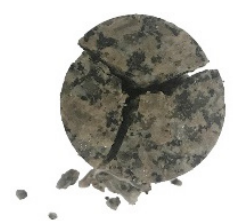

(d) $\mathrm{n}=1.2$

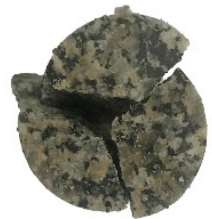

(e) $\mathrm{n}=1.4$

Figure 6. Failure modes of granite with different aspect ratios under $8 \mathrm{~m} / \mathrm{s} \mathrm{impact} \mathrm{velocity}$

\subsection{Analysis of energy-time density}

During the impact test, the energy lost by the contact interface between the pressure rod and the test piece is generally ignored. It is considered that the incident wave energy $E_{I}$ is completely converted into reflected wave energy $E_{R}$, transmitted wave energy $E_{T}$ and specimen dissipation energy $E_{D}$ :

$$
E_{\mathrm{I}}=E_{\mathrm{R}}+E_{\mathrm{T}}+E_{\mathrm{D}}
$$

The energy structure with time intensity is defined as energy-time density. This relationship can reveal the coupling law of rock energy absorption when the rock is broken, as is shown in equation (3). Since the time between the take-off and the end of the three waves is basically the same, the elapsed time of the three waves is taken as the stress wave acting time in the specimen.

$$
\begin{aligned}
& E_{\mathrm{V}}=E_{\mathrm{D}} / V_{\mathrm{S}} \\
& E_{\mathrm{VT}}=E_{\mathrm{D}} /\left(V_{\mathrm{S}} \cdot T_{\mathrm{R}}\right)
\end{aligned}
$$

In the formula: $E_{V}$ is the energy dissipation per unit volume; $E_{V T}$ is the energy-time density; $V_{S}$ is the volume of the specimen; $T_{R}$ is the reflected wave elapsed time. 

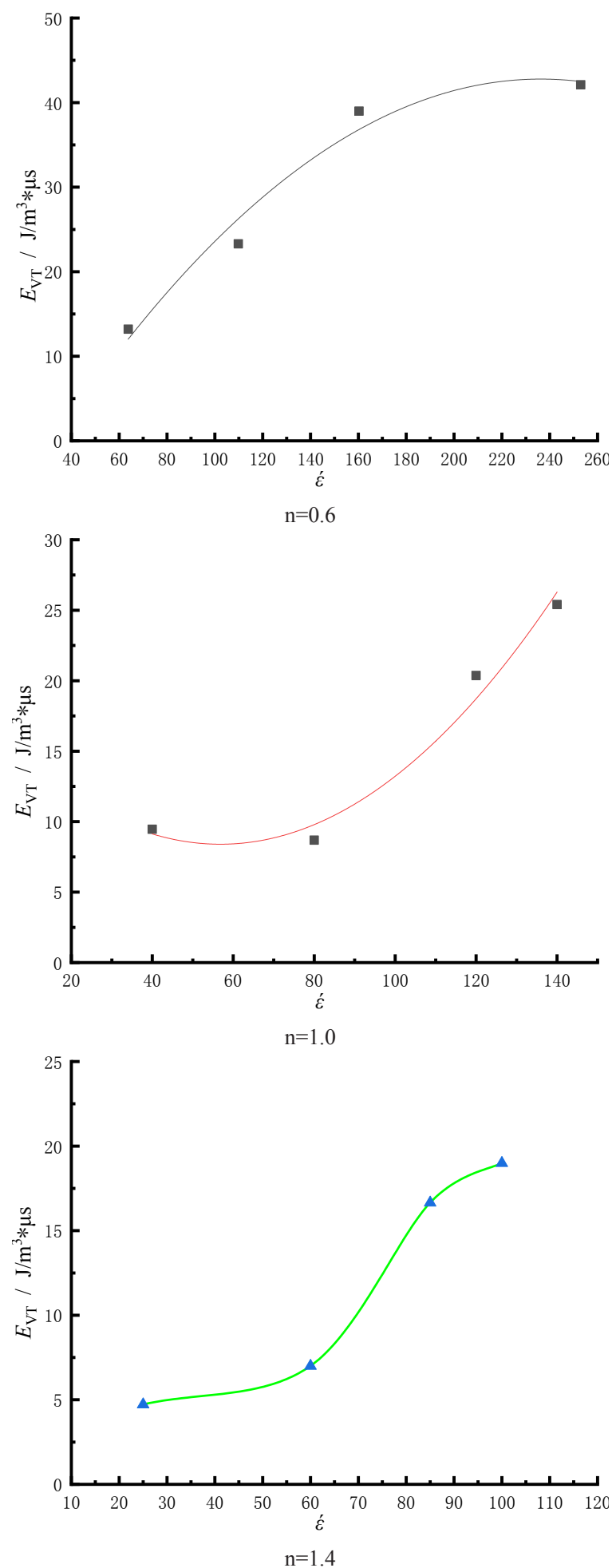

Figure 7. Relationship between energy-time density and strain rate of granites with different aspect ratios

When the load strain rate of the granite with an aspect ratio of 0.6 increases from $50 \mathrm{~s}^{-1}$ to $150 \mathrm{~s}^{-1}$, the energy-time density index increases significantly; when the load strain rate exceeds $150 \mathrm{~s}^{-1}$, the energy-time density index decreases. When the strain rate of granite with an aspect ratio of 1.0 exceeds $80 \mathrm{~s}^{-1}$, the energy-time density increases significantly. When the strain rate of granite with an aspect ratio of 1.4 exceeds $60 \mathrm{~s}^{-1}$, the energy-time density increase rate of the rock increases significantly. Comparing the energy-time density of the rock under the same loading strain rate, it can be found that the granite with an aspect ratio of 0.6 is easier to absorb the energy of the rock during the stress pulse time. The granite with an aspect ratio of 1.4 absorbs energy weakly during the stress pulse time. On the one hand, because the rock with a 
small aspect ratio is easier to reach the stress balance state, the stress balance time accounts for a relatively large amount of time within the stress pulse. However, the stress balance time of rocks with a large aspect ratio is shorter, and most of the rocks are in a state of stress deterioration, which reduces the energy-absorbing and crushing ability of the rock during the stress pulse.

\section{Conclusion}

(1) From the point of view of the microstructure characteristics of the stress-strain curve, granite originally belonged to the elastic compression stage, making the $\sigma-\varepsilon$ curve approximate to a linear rise. After the specimen stress rises to a certain value under different strain rates, the curve begins to become flat, that is, the slope of the curve gradually decreases. Before the stress of the specimen reaches the peak value, the curve fluctuates to varying degrees.

(2) The radial end face of the rock also cracks from the central area, and the stress concentration in the central area of the rock end face is faster than that in the boundary area. Under the action of a small loading strain rate, the rock is more likely to show a fractured shape. As the strain rate increases, the shape of the rock fracture changes from large to small.

(3) When the loading strain rate is between $100 \mathrm{~s}^{-1}$ and $150 \mathrm{~s}^{-1}$, the stress balance time is shortened, and the energy absorbed by the rock gradually increases during the stress pulse. In the process of large deformation and failure, the rock will inevitably cause deformation at both ends of the rock, which will degrade the stress balance. When the loading strain rate exceeds $150 \mathrm{~s}^{-1}$, the rock continues to absorb energy, but the excess energy absorbed will crush the rock excessively and reduce the energy utilization rate.

\section{References}

[1] Li X B, Wang S M, Weng L, et al. Damage constitutive model of different age concretes under impact load. Joumal of Central South University. 2015; 22(2): 693-700.

[2] Li Xibing, Tao Ming, Gong Fengqiang, et al. Theoretical and experimental study of hard rock spalling fracture under impact dynamic loading. Chinese Journal of Rock Mechanics and Engineering. 2011; 30(6): 1081-1088.

[3] Li Diyuan, Cheng Tengjiao, Zhou Tao, et al. Experimental study of the dynamic strength and fracturing characteristics of marble specimens with a single hole under impact loading. Chinese Journal of Rock Mechanics and Engineering. 2015; 34(2): 249-260.

[4] Ping Qi, Luo Xuan, Ma Qinyong, et al. Fragmentation energy consumption characteristics of sandstone specimens under impact load. Chinese Journal of Rock Mechanics and Engineering. 2015; 34(S2): 4197-4203.

[5] Liu Xiaohui, Zhang Ru, Liu Jianfeng, Experimental study on impact dynamics of coal and rock under different strain rates. Journal of China Coal Society. 2012; 37(9): 1528-1534.

[6] Liu Xiaohui, Zhang Ru, Liu Jianfeng, Experimental study on the relationship between coal fragmentation and energy consumption under impact loading. China Coal. 2014; 40(6): 45-49.

[7] Li Yexue, Liu Jianfeng, Qin Li. Experimental study on the law of energy dissipation when stress waves pass through rock joints. Experimental Mechanics. 2011; 26(1): 85-90.

[8] Li Yexue, Peng Qi, Zhu Jianbo, et al. Experimental study on the influence of fractal intercept on stress wave energy consumption. Chinese Journal of Rock Mechanics and Engineering. 2011; 30(S2): 3982-3988.

[9] Liang Changyu, Li Xiao, Zhang Hui, et al. Study on size effect of uniaxial compression characteristics of granite in the range of low and medium strain rates. Chinese Journal of Rock Mechanics and Engineering. 2013; 32(3): 528536.

[10] Li Q M. Strain energy density failure criterion. International Journal of Solids and Structures. 2001; 38: 6997-7013.

[11] Zhao Guoyan, Li Zhenyang, Wu Hao, et al. Research on dynamic failure characteristics of sandstone with discontinuous fissures. Rock and Soil Mechanics. 2019; 40(S1): 73-81.

[12] Guo Lianjun, Yang Yuehui, Zhang Daning, Li Lin. Energy consumption analysis of magnetite quartzite crushing under impact load. Metal Mine. 2014; (08): 1-5.

[13] Gong Fengqiang, Li Xibing, Rao Qiuhua, Liu Xiling. Reference method for determining the sample size in rock SHPB test. Vibration and Shock. 2013; 32(17): 24-28.

[14] Xia K, Nasseri MHB, Mohanty B, Lu F, Chen R, Luo S N. Effects of microstructures on dynamic compression of barre granite. International Journal of Rock Mechanics \& Mining Sciences. 2008; 45(6): 879-887. 